\title{
WEAK NON-ASSOCIATIVE STRUCTURES OF GROUPS WITH APPLICATIONS
}

\author{
SHAH NAWAZ ${ }^{1}$, MUHAMMAD GULISTAN ${ }^{1}$, NAVEED YAQOOB ${ }^{2}$ AND SEIFEDINE \\ $\mathrm{KADRY}^{3, *}$ \\ ${ }^{1}$ Department of Mathematics, Hazara University, Mansehra, KP, Pakistan \\ ${ }^{2}$ Department of Mathematics, College of science, Al-Zulfi Majmaah University, Al-Zulfi Saudi Arabia \\ ${ }^{3}$ Department of Mathematics and Computer Science, Faculty of Science, Beirut Arab University, P.O. Box \\ 11-5020, Beirut 11072809, Lebanon
}

*Corresponding author: s.kadry@bau.edu.lb

\begin{abstract}
Inspiring by the weak symmetry occurring in the $\mathcal{H}_{v}$-left invertive structures, in this article we have introduce a new class of $\mathcal{H}_{v}-\mathcal{L} \mathcal{A}$-groups which is a generalization of $\mathcal{L} \mathcal{A}$-hypergroups. We have investigated different types of homomorphisms of $\mathcal{H}_{v}-\mathcal{L} \mathcal{A}$-groups. Moreover, we have constructed the $\mathcal{H}_{v^{-}}$ $\mathcal{L} \mathcal{A}$-groups. At the end a useful application of weak symmetry related with $\mathcal{H}_{v}$-left invertive structure has been presented using the chemical redox reaction.
\end{abstract}

\section{INTRODUCTION}

Kazim and naseerudin [1] laid the idea of left almost semigroup (denoted by LA-semigroups). They generalized some handy result of semigroup theory. Afterwards, Mushtaq [2] and other, went further in the detail of the structure and added various beneficial results to the theory of $\mathcal{L} \mathcal{A}$-semigroup, see paper [3-9]. An $\mathcal{L} \mathcal{A}$-semigroup is midway structure between commutative semigroup and groupoid. Mushtaq and kamran [10] in 1996 proposed the idea of left almost groups. They proved that if $G$ is left almost group and $H$ is left

Received 2019-05-15; accepted 2019-06-19; published 2019-09-02.

2010 Mathematics Subject Classification. 20 N05.

Key words and phrases. hyperoperation; $\mathcal{H}_{v}$ - $\mathcal{L} \mathcal{A}$-groups; homomorphisms; chemical redox reaction.

(C)2019 Authors retain the copyrights of their papers, and all open access articles are distributed under the terms of the Creative Commons Attribution License. 
almost subgroup then $G / H$ is a set of left almost group. Hyperstucture notion was initiated by Marty in 1934, when he [11] elucidated hypergroup and embarked in analyze their properties and applied them to a group. Handful papers and books have been written in this direction, see [12-14]. In 1990, in Greece, Thomas Vougiouklis was organized a congress on hyperstructure and name it AHA, but also there had been three more congresses organized in Italy by Corsini, on the same topic with different name. During that congress Vougiouklis [17] introduced the concept of weak structure which is now named $\mathcal{H}_{v}$-structures. The impetus of $\mathcal{H}_{v}$-structures is that, the quotient of group with respect to invariant subgroup is a group. Marty from 1934 notify that, the quotient of group with respect to any subgroup is a hypergroup. Finally, the quotient of a group with respect to any partition (equivalently to any equivalence relation) is an $\mathcal{H}_{v}$-group. Let $(G, \circ)$ be group and $R$ be equivalence relation, then $(G / R, \circ)$ is an $\mathcal{H}_{v}$-Group. Several authors have studied different aspects of $\mathcal{H}_{v}$-structure. For instance, Vougiouklis [18, 19], Spratalis [20-24] and Davvaz [25]. Not long ago, in 2011, Hila and Dine [15] laid the idea of LA-semihypergroups, which is generalization of semigroups, semihypergroups and $\mathcal{L} \mathcal{A}$-semigroups. Yaqoob, Corsini and Yousfzai [16] extended the work of Hila and Dine and characterized intera regular left almost hypergroups by their hyperideal by using pure left identity. The idea of $\mathcal{H}_{v}-\mathcal{L} \mathcal{A}$-semigroups was given by Gulistan et al., [26] in 2015. Another motivation for the study of hyperstructure comes from chemical reaction, such as chain redox and dismutation reaction were provided different example of weak structures. for detail see papers [27-29].

This very article communicates the novel class of $\mathcal{H}_{v}-\mathcal{L} \mathcal{A}$-groups which is a generalization of $\mathcal{L} \mathcal{A}$-hypergroups. We have defined various types of homomorphisms. Additionally, we have constructed the $\mathcal{H}_{v}-\mathcal{L} \mathcal{A}$-groups, and presented the chemical example by making use of redox reactions.

\section{Preliminaries}

In this section we recall some helping material from different papers, like $[16,19,26]$.

Definition 2.1. [16] A hypergroupoid $(\mathcal{H}, \circ)$ is called $\mathcal{L} \mathcal{A}$-semihypergroup, if it satisfies the following law

$$
(x \circ y) \circ z=(z \circ y) \circ x \text { for all } x, y, z \in \mathcal{H}
$$

Example: [16] Let $\mathcal{H}=Z$ if we define $x \circ y=y-x+3 Z$, where $x, y \in Z$. Then $(\mathcal{H}, \circ)$ becomes an $\mathcal{L} \mathcal{A}$-semihypergroup.

Definition 2.2. [19] The hyperoperation $*: \mathcal{H} \times \mathcal{H} \longrightarrow P^{*}(\mathcal{H})$ is called weakly associative hyperoperation (abbreviated as $W A S S$ ) if for any $a, b, c \in \mathcal{H}$

$$
(a * b) * c \cap a *(b * c) \neq \emptyset
$$

Definition 2.3. [19] The hyperoperation is weakly commutative (abbreviated as COW) if for any a,b $\in \mathcal{H}$

$$
a * b \cap b * a \neq \phi
$$


Definition 2.4. [26] Let $\mathcal{H}$ be non-empty set and $*$ be hyperoperation on $\mathcal{H}$. Then $(\mathcal{H}, *)$ is called an $\mathcal{H}_{v}-\mathcal{L} \mathcal{A}$-semigroup, if it satisfies the weak left invertive law, for all $x, y, z \in \mathcal{H}$

$$
(x * y) * z \cap(z * y) * x \neq \emptyset
$$

Example: [26] Let $\mathcal{H}=(0, \infty)$ we define $x * y=\left\{\frac{y}{x+1}, \frac{y}{x}\right\}$ where $x, y \in \mathcal{H}$. Then for all $x, y, z \in \mathcal{H}$ satisfies $(x * y) * z \cap(z * y) * x \neq \emptyset$. Hence $(\mathcal{H}, *)$ is an $\mathcal{H}_{v}-\mathcal{L} \mathcal{A}$-semigroup.

\section{3. $\mathcal{H}_{v}-\mathcal{L} \mathcal{A}$-Groups}

In this section, we present a new generalize class of non-associative hyperstructures namely $\mathrm{H}_{v}$ - $\mathcal{L} \mathcal{A}$-groups and provided different examples and some basic results.

Definition 3.1. Let $\mathcal{H}$ be a non-empty set and $*$ be hyperoperation on $\mathcal{H}$. Then $(\mathcal{H}, *)$ is called an $\mathcal{H}_{v}-\mathcal{L} \mathcal{A}$ group, if it satisfies the following axioms

(i) $(x * y) * z \cap(z * y) * x \neq \emptyset$ for all $x, y, z \in \mathcal{H}$,

(ii) $\mathcal{H} * x=\mathcal{H}=\mathcal{H} * x$ for all $x \in \mathcal{H}$.

Example: Let $\mathcal{H}=\{a, b, c\}$ be a finite set. The hyperoperation $*$ is defined as follow

\begin{tabular}{|l|l|l|l|}
\hline$*$ & $a$ & $b$ & $c$ \\
\hline$a$ & $a$ & $\{a, b\}$ & $\{a, c\}$ \\
\hline$b$ & $c$ & $\{\mathcal{H}\}$ & $\{a, b\}$ \\
\hline$c$ & $b$ & $\{a, c\}$ & $\{\mathcal{H}\}$ \\
\hline
\end{tabular}

Here all elements of $\mathcal{H}$ satisfy the weak left invertive law. Also left invertive law is not hold in $\mathcal{H}$, i.e.

$$
\mathcal{H}=(a * b) * c \neq(c * b) * a=\{a, b\}
$$

Alike, associative law is not hold in $\mathcal{H}$, i.e.

$$
\mathcal{H}=(c * c) * a \neq c *(c * a)=\{a, c\} .
$$

Even, weak associative law is not true

$$
\{b\}=(b * a) * a \cap b *(a * a)=\{c\}=\phi
$$

Hence $(\mathcal{H}, *)$ is an $\mathcal{H}_{v}-\mathcal{L} \mathcal{A}$-group. 
Example: Let $\mathcal{H}=\{a, b, c, d\}$ be a finite set and the hyperoperation is defined in the following table.

\begin{tabular}{|l|l|l|l|l|}
\hline$*$ & $a$ & $b$ & $c$ & $d$ \\
\hline$a$ & $a$ & $b$ & $c$ & $d$ \\
\hline$b$ & $c$ & $\{a, c\}$ & $\{b, c\}$ & $d$ \\
\hline$c$ & $b$ & $\{a, c\}$ & $\{a, c\}$ & $d$ \\
\hline$d$ & $d$ & $d$ & $d$ & $\{a, b, c\}$ \\
\hline
\end{tabular}

As all elements of $\mathcal{H}$ satisfy the weak left invertive law but $\mathcal{H}$ do not satisfies the left invertive law, associative law and weak associative law i.e.

$$
\begin{aligned}
\{a, c\} & =(b * b) * c \neq(c * b) * b=\{a, b, c\}, \\
\text { and }\{a, c\} & =(b * b) * c \neq b *(b * c)=\{a, b, c\} . \\
\text { Also }\{b\} & =(b * a) * a \cap b *(a * a)=\{c\}=\phi
\end{aligned}
$$

So $(\mathcal{H}, *)$ is an $\mathcal{H}_{v}-\mathcal{L} \mathcal{A}$-group.

Now we will discuss some very basic results related with $\mathcal{H}_{v}-\mathcal{L} \mathcal{A}$-groups.

Lemma 3.1. If $(\mathcal{H}, *)$ is an $\mathcal{H}_{v}-\mathcal{L} \mathcal{A}$-group, then

$$
(a * b) *(c * d) \cap(a * c) *(b * d) \neq \phi, \text { hold for all } a, b, c, d \in \mathcal{H}
$$

Proof: Let us consider

$$
\begin{aligned}
x & \in(a * b) *(c * d) \\
& =((c * d) * b) * a \\
& =((b * d) * c) * a \\
& =(a * c) *(b * d) .
\end{aligned}
$$

This implies that $x \in(a * c) *(b * d)$. From this we can say that $(a * b) *(c * d) \cap(a * c) *(b * d) \neq \phi$, hold for all $a, b, c, d \in \mathcal{H}$.

Proposition 3.1. Let $(\mathcal{H}, \circ)$ be an $\mathcal{L} \mathcal{A}$-hypergroup with left identity e and non-empty set $A$, such that $A \subseteq H$. If $(A \circ(A \circ x)) \circ y \cap(A \circ(A \circ y)) \circ x \neq \emptyset \forall x, y \in \mathcal{H}$ and we define a hyperoperation $A_{R}^{\otimes}$ on $\mathcal{H}$ as $x A_{R}^{\otimes} y=(x \circ y) \circ A$, then $\left(\mathcal{H}, A_{R}^{\otimes}\right)$ become an $\mathcal{H}_{v}-\mathcal{L} \mathcal{A}$-group. 
Proof: Let $x, y, z \in \mathcal{H}$, we have

$$
\begin{aligned}
\left(x A_{R}^{\otimes} y\right) A_{R}^{\otimes} z & =((x \circ y) \circ A) A_{R}^{\otimes} z \\
& =(((x \circ y) \circ A) \circ z) \circ A \\
& =((z \circ A) \circ(x \circ y)) \circ A \\
& =(A \circ(A \circ z)) \circ(y \circ x) \\
& =y \circ((A \circ(A \circ z)) \circ x)
\end{aligned}
$$

and on the other hand

$$
\begin{aligned}
\left(z A_{R}^{\otimes} y\right) A_{R}^{\otimes} x & =((z \circ y) \circ A) A_{R}^{\otimes} x \\
& =(((z \circ y) \circ A) \circ z) \circ A \\
& =((x \circ A) \circ(z \circ y)) \circ A \\
& =(A \circ(A \circ x)) \circ(y \circ z) \\
& =y \circ((A \circ(A \circ x)) \circ z)
\end{aligned}
$$

but, since $y \circ((A \circ(A \circ z)) \circ x) \cap y \circ((A \circ(A \circ x)) \circ z) \neq \emptyset \forall$ for all $x, y, z \in \mathcal{H}$. It follows that $\left(x A_{R}^{\otimes} y\right) A_{R}^{\otimes} z \cap$ $\left(z A_{R}^{\otimes} y\right) A_{R}^{\otimes} x \neq \emptyset$. Next, we have

$$
x A_{R}^{\otimes} \mathcal{H}=(x \circ \mathcal{H}) \circ A=\mathcal{H} \text { also } \mathcal{H} A_{R}^{\otimes} x=(\mathcal{H} \circ x) \circ A=\mathcal{H} .
$$

Hence $\left(\mathcal{H}, A_{R}^{\otimes}\right)$ becomes an $\mathcal{H}_{v}-\mathcal{L} \mathcal{A}$-group.

Next we defined some regular relations on $\mathcal{H}_{v}-\mathcal{L} \mathcal{A}$-groups.

Definition 3.2. The equivalence relation $\rho$ is called regular on right (on the left), if for all $x$ of $\mathcal{H}$ ( $\mathcal{H}$ is an $\mathcal{H}_{v}-\mathcal{L} \mathcal{A}$-group $)$, from $a \rho b$, it follows that $(a \circ x) \bar{\rho}(b \circ x),(x \circ a) \bar{\rho}(x \circ b)$ respectively.

Lemma 3.2. The relation $\rho=\phi^{-1} * \phi=\left\{(x, y) \in \mathcal{H}_{1} \times \mathcal{H}_{1}: \phi(x)=\phi(y)\right\}$ is regular on $\mathcal{H}_{1}$.

Proof: The $\rho$ is an equivalence relation on $\mathcal{H}_{1}$ obviously. We have to show that $\rho$ is regular on $\mathcal{H}_{1}$. Let $x, y, z \in \mathcal{H}_{1}$ such that $x \rho y$, this implies that $\phi(x)=\phi(y) \Longrightarrow \phi(x z)=\phi(y z)$ and $\phi(z x)=\phi(z y)$. so $(x z) \rho(y z)$. Thus $x \rho y \Longrightarrow(x z) \rho(y z)$ and $(z x) \rho(z y)$. Hence $\rho=\phi^{-1} * \phi=\left\{(x, y) \in \mathcal{H}_{1} \times \mathcal{H}_{1}: \phi(x)=\phi(y)\right\}$ is regular on $\mathcal{H}_{1}$.

On $\mathcal{H}_{v}-\mathcal{L} \mathcal{A}$-group $\mathcal{H}$, we are concerned with equivalence relation for which the family of equivalence classes form an $\mathcal{H}_{v}-\mathcal{L} \mathcal{A}$-group under the hyperoperation induced by that on $\mathcal{H}$. For an equivalence relation $\rho$ on $\mathcal{H}$, we may use $x_{\rho}$, and $\bar{x}$ or $\rho(x)$ to denote the equivalence class of $x \in \mathcal{H}$. Moreover, generally if $A$ is a 
non-empty subset of $\mathcal{H}$ then $A_{\rho}=U\left\{x_{\rho} \mid x \in A\right\}$. We let $\mathcal{H} / \rho$ denote the family $\left\{x_{\rho} \mid x \in \mathcal{H}\right\}$ of class of $\rho$. The hyperoperation on $\mathcal{H}$ induces a hyperoperation $\otimes$ on $\mathcal{H} / \rho$ defined by

$$
x_{\rho} \otimes y_{\rho}=\left\{z_{\rho} / z \in x_{\rho} \circ y_{\rho}\right\}
$$

Where $x, y \in \mathcal{H}$. The structure $(\mathcal{H} / \rho, \otimes)$ is known as quotient structure.

Theorem 3.1. Let $(\mathcal{H}, \circ)$ be $\mathcal{H}_{v}-\mathcal{L} \mathcal{A}$-group. Then $(\mathcal{H} / \rho, \otimes)$ is an $\mathcal{H}_{v}-\mathcal{L} \mathcal{A}$-group iff

$$
(\rho(x) \circ \rho(y)) \circ \rho(z) \cap(\rho(z) \circ \rho(y)) \circ \rho(x) \neq \phi \forall x, y, z \in \mathcal{H}
$$

Proof: In $\mathcal{H} / \rho$, we have

$$
\text { let } \begin{aligned}
U & \in(\rho(x) \otimes \rho(y)) \otimes \rho(z)=\{u / u \in x \circ y\} \otimes z \\
& =\{t \in u \circ z, u \in x \circ y\} \\
& =\{t \in(z \circ y) \circ x\} \\
& \Longrightarrow U \in(\rho(x) \otimes \rho(y)) \otimes \rho(z)
\end{aligned}
$$

This implies that $(\rho(x) \otimes \rho(y)) \otimes \rho(z) \subseteq(\rho(z) \otimes \rho(y)) \otimes \rho(x)$. So $(\rho(x) \otimes \rho(y)) \otimes \rho(z) \cap(\rho(z) \otimes \rho(y)) \otimes$ $\rho(x) \neq \phi$. Since $x \circ \mathcal{H}=\mathcal{H}=\mathcal{H} \circ x, \Longrightarrow$ whence $\mathcal{H} / \rho \otimes \bar{x}=\mathcal{H} / \rho=\bar{x} \otimes \mathcal{H} / \rho$. Hence $(\mathcal{H} / \rho, \otimes)$ is an $\mathcal{H}_{v}$-LA-group.

Theorem 3.2. Let $(\mathcal{H}, \circ)$ be $\mathcal{H}_{v}-\mathcal{L} \mathcal{A}$-group and $\rho$ be equivalence relation on $\mathcal{H}$. If $\rho$ is a regular relation, then $(\mathcal{H} / \rho, \otimes)$ is an $\mathcal{H}_{v}-\mathcal{L} \mathcal{A}$-group.

Proof: First we show that $\otimes$ is a well defined on $\mathcal{H} / \rho$, consider $\bar{x}=\bar{x}_{1}$ and $\bar{y}=\bar{y}_{1}$. We check that $\bar{x} \otimes \bar{y}=\bar{x}_{1} \otimes \bar{y}_{1}$. We have $x \rho x_{1}$ and $y \rho y_{1}$. Since $\rho$ is regular, it follows that $(x \circ y) \bar{\rho}\left(x_{1} \circ y\right),\left(x_{1} \circ y\right) \bar{\rho}\left(x_{1} \circ y_{1}\right)$ whence $(x \circ y) \bar{\rho}\left(x_{1} \circ y_{1}\right)$. This implies that for all $n \in(x \circ y)$ there exists $n_{1}$ such that $n \rho n_{1}$. Which shows that $\bar{n}=\bar{n}_{1}$. It follows that that $\bar{x} \otimes \bar{y} \subseteq \bar{x}_{1} \otimes \bar{y}_{1}$ and similarly we obtain converse. Hence $\otimes$ is well defined . Next we show weak left invertive property of $\otimes$. Let $\bar{x}, \bar{y}, \bar{z}$ be arbitrary element in $\mathcal{H} / \rho$ and $\bar{l} \in(\bar{x} \otimes \bar{y}) \otimes \bar{z}$. This implies that $\bar{v} \in \bar{x} \otimes \bar{y}$ and $\bar{l} \in \bar{v} \otimes \bar{z}$. It means that $v_{1} \in x \circ y$ and $l_{1} \in v \circ z$ such that $v \rho v_{1}$ and $l \rho l_{1}$. since $\rho$ is regular relation, it follows that there exists $l_{2} \in v_{1} \circ z \subseteq(x \circ y) \circ z \subseteq(z \circ y) \circ x$ ( since $\mathcal{H}$ is a $\mathcal{H}_{v}$-LA-group) such that $l_{1} \rho l_{2}$. From here we obtain that there exist $l_{3} \in z \circ y$ such that $l_{2} \in l_{3} \circ x$. we have

$$
\begin{gathered}
\bar{l} \quad=\quad \bar{l}_{1}=\bar{l}_{2} \in \bar{l}_{3} \otimes \bar{x} \subseteq(\bar{z} \otimes \bar{y}) \otimes \bar{x} \\
\Longrightarrow \quad(\bar{x} \otimes \bar{y}) \otimes \bar{z} \subseteq(\bar{z} \otimes \bar{y}) \otimes \bar{x} \\
\Longrightarrow \quad(\bar{x} \otimes \bar{y}) \otimes \bar{z} \cap(\bar{z} \otimes \bar{y}) \otimes \bar{x} \neq \phi
\end{gathered}
$$


Finally we show the reproductive axiom

$$
\begin{aligned}
\text { since } x \circ \mathcal{H} & =\mathcal{H}=\mathcal{H} \circ x \\
\text { whence } \bar{x} \otimes \mathcal{H} / \rho & =\mathcal{H} / \rho=\mathcal{H} / \rho \otimes \bar{x} .
\end{aligned}
$$

Hence $(\mathcal{H} / \rho, \otimes)$ is a $\mathcal{H}_{v}$-LA-group.

Next we defined the homorphisms of $\mathcal{H}_{v}$-LA-groups.

Definition 3.3. A mapping $\phi: \mathcal{H}_{1} \longrightarrow \mathcal{H}_{2}$ (where $H_{1}$ and $H_{2}$ are $\mathcal{H}_{v}$-LA-group $)$ is said to be good homomorphism if it satisfies the following property

$$
\phi(x y)=\phi(x) \phi(y) \forall x, y \in \mathcal{H}_{1}
$$

Example: Let $\mathcal{H}_{1}=\{a, b, c\}$ and $\mathcal{H}_{2}=\{l, m, n\}$, be two $\mathcal{H}_{v}$-LA-hypergroups with hyperoperation is defined in the following tables respectively,

\begin{tabular}{|l|l|l|l|}
\hline$*$ & $l$ & $m$ & $n$ \\
\hline$l$ & $\{l\}$ & $\{m\}$ & $\{n\}$ \\
\hline$m$ & $\{n\}$ & $\{l, n\}$ & $\{m\}$ \\
\hline$n$ & $\{m\}$ & $\{l, n\}$ & $\{l, n\}$ \\
\hline
\end{tabular}

and

\begin{tabular}{|l|l|l|l|}
\hline$\circ$ & $a$ & $b$ & $c$ \\
\hline$a$ & $\{a\}$ & $\{b\}$ & $\{c\}$ \\
\hline$b$ & $\{c\}$ & $\{a, c\}$ & $\{b, c\}$ \\
\hline$c$ & $\{b\}$ & $\{a, c\}$ & $\{a, c\}$ \\
\hline
\end{tabular}

The mapping $f: \mathcal{H}_{1} \longrightarrow \mathcal{H}_{2}$ is defined by $f(a)=l, f(b)=m, f(c)=n$. Then assuredly $f$ homomorphism is a strong homomorphism.

If good homomorphism is $1-1$ and onto is called isomorphism. If $f$ is an isomorphism, then $\mathcal{H}_{1}$ and $\mathcal{H}_{2}$ are said to be isomorphic, which is denoted by $H_{1} \cong \mathcal{H}_{2}$.

Definition 3.4. Let $\left(\mathcal{H}_{1}, \circ\right)$ and $\left(\mathcal{H}_{2}, *\right)$ be two $\mathcal{H}_{v}$ - $\mathcal{L} \mathcal{A}$-hypergroups. The map $f: \mathcal{H}_{1} \longrightarrow \mathcal{H}_{2}$ is called inclusion homomorphism if for all $x, y \in \mathcal{H}_{1}$ satisfies the following property

$$
f(x \circ y) \subseteq f(x) * f(y)
$$


Example: Let $\mathcal{H}_{1}=\{l, m, n\}$ and $\mathcal{H}_{2}=\{a, b, c\}$ be two $\mathcal{H}_{v}$-LA- hypergroups with hyperoperations defined in the following table:

\begin{tabular}{|l|l|l|l|}
\hline$*$ & $l$ & $m$ & $n$ \\
\hline$l$ & $\{l\}$ & $\{m\}$ & $\{n\}$ \\
\hline$m$ & $\{n\}$ & $\{l, m\}$ & $\{m\}$ \\
\hline$n$ & $\{m\}$ & $\{n\}$ & $\{n, l\}$ \\
\hline
\end{tabular}

and

\begin{tabular}{|l|l|l|l|}
\hline$\circ$ & $a$ & $b$ & $c$ \\
\hline$a$ & $\{a\}$ & $\{b\}$ & $\{c\}$ \\
\hline$b$ & $\{c\}$ & $\{\mathcal{H}\}$ & $\{b\}$ \\
\hline$c$ & $\{b\}$ & $\{c\}$ & $\{\mathcal{H}\}$ \\
\hline
\end{tabular}

The mapping $f: \mathcal{H}_{1} \longrightarrow \mathcal{H}_{2}$ is defined by $f(l)=a, f(m)=b, f(n)=c$. Then clearly $f$ is an inclusion homomorphism.

Definition 3.5. Let $\left(\mathcal{H}_{1}, \circ\right)$ and $\left(\mathcal{H}_{2}, *\right)$ be two $\mathcal{H}_{v}$ - $\mathcal{L} \mathcal{A}$-hypergroup. The map $f: \mathcal{H}_{1} \longrightarrow \mathcal{H}_{2}$ is called weak homomorphism or $\mathcal{H}_{v}$ homomorphism, if for all $x, y \in \mathcal{H}_{1}$, the condition is hold

$$
f(x \circ y) \cap f(x) * f(y) \neq \phi
$$

Example: Let $\mathcal{H}_{1}=\{l, m, n\}$ and $\mathcal{H}_{2}=\{a, b, c\}$ are two finite sets, where $\left(\mathcal{H}_{1}, *\right)$ and $\left(\mathcal{H}_{2}, \circ\right)$ are $\mathcal{H}_{v}$-LA-hypergroups, the hyperoperation is defined in following tables:

\begin{tabular}{|l|l|l|l|}
\hline$*$ & $l$ & $m$ & $n$ \\
\hline$l$ & $\{l\}$ & $\{m\}$ & $\{n\}$ \\
\hline$m$ & $\{n\}$ & $\{l, m\}$ & $\{m\}$ \\
\hline$n$ & $\{m\}$ & $\{n\}$ & $\{n, l\}$ \\
\hline
\end{tabular}

and

\begin{tabular}{|l|l|l|l|}
\hline$\circ$ & $a$ & $b$ & $c$ \\
\hline$a$ & $a$ & $\{a, b\}$ & $\{a, c\}$ \\
\hline$b$ & $c$ & $\{\mathcal{H}\}$ & $\{a, b\}$ \\
\hline$c$ & $b$ & $\{a, c\}$ & $\{\mathcal{H}\}$ \\
\hline
\end{tabular}

The mapping $f: \mathcal{H}_{1} \longrightarrow \mathcal{H}_{2}$ is defined by $f(l)=a, f(m)=b, f(n)=c$. Then clearly $f$ is a weak homomorphism or $\mathcal{H}_{v}$-LA-homomorphism.

Theorem 3.3. Let $\phi: \mathcal{H}_{1} \longrightarrow \mathcal{H}_{2}$ be good homomorphism of an $\mathcal{H}_{v}-\mathcal{L} \mathcal{A}$ groups. Then there exist a monomorphism $\psi: \mathcal{H}_{1} / \rho \longrightarrow \mathcal{H}_{2}$ such that $i m \phi=i m \psi$ and diagram 


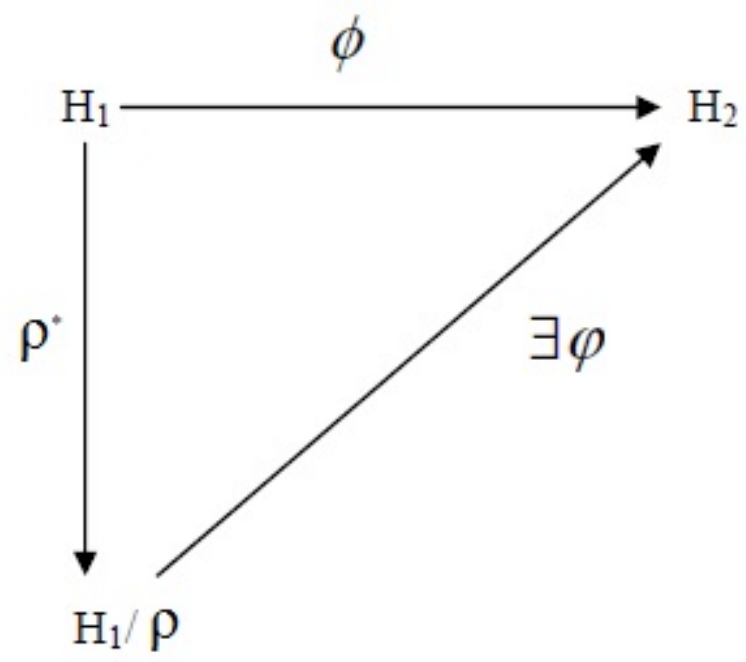

commutes i.e. $\psi * \rho^{\bullet}=\phi$ where the mapping $\rho^{\bullet}: \mathcal{H}_{1} \longrightarrow \mathcal{H}_{1} / \rho$ is defined by $\rho^{\bullet}(x)=\rho(x) \forall x \in \mathcal{H}_{1}$.

Proof: Let $\psi: \mathcal{H}_{1} / \rho \longrightarrow \mathcal{H}_{2}$ is defined as $\psi(\rho(x))=\phi(x) \forall x \in \mathcal{H}_{1}$ since $\phi: \mathcal{H}_{1} \longrightarrow \mathcal{H}_{2}$. First we show that $\psi$ is well defined. For this let $\rho\left(x_{1}\right), \rho\left(x_{2}\right) \in \mathcal{H}_{1} / \rho$ such that

$$
\begin{aligned}
\rho\left(x_{1}\right) & =\rho\left(x_{2}\right) \\
\rho^{\bullet}\left(x_{1}\right) & =\rho^{\bullet}\left(x_{2}\right) \\
\psi\left(\rho^{\bullet}\left(x_{1}\right)\right) & =\psi\left(\rho^{\bullet}\left(x_{2}\right)\right) \\
\phi\left(x_{1}\right) & =\phi\left(x_{2}\right) \\
\psi\left(\rho\left(x_{1}\right)\right) & =\psi\left(\rho\left(x_{2}\right)\right) .
\end{aligned}
$$

Next we will show that $\psi$ is one -one. For this let $\psi\left(\rho\left(x_{1}\right)\right), \psi\left(\rho\left(x_{2}\right)\right) \in \mathcal{H}_{1} / \rho$

$$
\begin{aligned}
\psi\left(\rho\left(x_{1}\right)\right) & =\psi\left(\rho\left(x_{2}\right)\right) \\
\phi\left(x_{1}\right) & =\phi\left(x_{2}\right) \\
\psi\left(\rho^{\bullet}\left(x_{1}\right)\right) & =\psi\left(\rho^{\bullet}\left(x_{2}\right)\right) \\
\rho^{\bullet}\left(x_{1}\right) & =\rho^{\bullet}\left(x_{2}\right) \\
\rho\left(x_{1}\right) & =\rho\left(x_{2}\right) .
\end{aligned}
$$

Finally we show that $\psi$ is homomorphism. Let $x, y \in \mathcal{H}_{1}$ we have

$$
\begin{aligned}
\psi(\rho(x) * \rho(y)) & =\{\psi(\rho(z)): z \in x y\}=\{\phi(z): z \in x y\} \\
& =\phi(x y)=\phi(x) \phi(y)=\psi(\rho(x)) * \psi(\rho(y))
\end{aligned}
$$


Hence $\psi$ is monomorphism and it is easy to prove that $i m \phi=i m \psi$. Now for all $x \in \mathcal{H}_{1}$, we have $\left(\psi * \rho^{\bullet}\right)(x)=$ $\psi\left(\left(\rho^{\bullet}\right)(x)\right)=\psi((\rho)(x))=\phi(x)$. Hence diagram commutes.

Theorem 3.4. Let $\phi: \mathcal{H}_{1} \longrightarrow \mathcal{H}_{2}$ be good homomorphism of an $\mathcal{H}_{v}-$ LA groups. Then $\mathcal{H}_{1} / \rho \cong \operatorname{Im} \phi$.

Proof: It follows from the Theorem 3.3.

Theorem 3.5. Let $\phi: \mathcal{H}_{1} \longrightarrow \mathcal{H}_{2}$ be good homomorphism of an $\mathcal{H}_{v}-\mathcal{L} \mathcal{A}$ groups. If $k$ is a regular relation on $\mathcal{H}_{1}$ such that $k \subseteq \rho$, then there exists a unique monomorphism $\psi: \mathcal{H}_{1} / k \longrightarrow \mathcal{H}_{2}$ such that $\operatorname{Im} \phi=\operatorname{Im} \psi$ and diagram

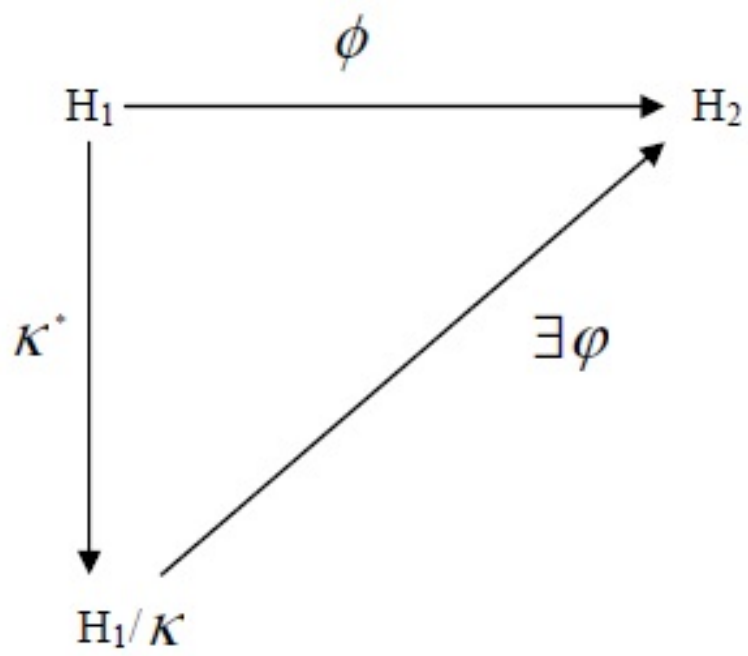

commute i.e. $\psi * k^{\star}=\phi$, where mapping $k^{\star}: \mathcal{H}_{1} \longrightarrow \mathcal{H}_{2} / k$ is defined as $k^{\star}(x)=k(x) \forall x \in \mathcal{H}_{1}$.

Proof: Straightforward.

Theorem 3.6. Let $\theta$ and $\sigma$ be two regular relation on an $\mathcal{H}_{v}-\mathcal{L} \mathcal{A}$-group $\mathcal{H}$ such that $\theta \subseteq \sigma$. Then $\sigma / \theta$ is regular relation on $\mathcal{H} / \theta$.

Proof: We define $\sigma / \theta: \mathcal{H} / \theta \circ \mathcal{H} / \theta \longrightarrow P^{\star}(\mathcal{H} / \theta)$ by $\sigma / \theta(\theta(x))=\theta(x) \forall \theta(x) \in \mathcal{H} / \theta$. We first show that the mapping is well defined, consider $\theta(x)=\theta(y) \Longrightarrow(x, y) \in \theta \subseteq \sigma \Longrightarrow(\theta(x), \theta(y)) \in \sigma / \theta$ and so $\sigma / \theta(\theta(x))=\sigma / \theta(\theta(y))$. Next we show that $\sigma / \theta$ is an equivalence relation. Let $x \in \mathcal{H}$, then $(x, x) \in \sigma \Longrightarrow(\theta(x), \theta(y)) \in \sigma / \theta$, thus $\sigma / \theta$ is reflexive. Also let $x, y \in \mathcal{H}$, such that $(\theta(x), \theta(y)) \in \sigma / \theta$. As $(x, y) \in \sigma \Longrightarrow(y, x) \in \sigma$ due to the symmetry of $\sigma$. Which implies that $(\theta(y), \theta(x)) \in \sigma / \theta$. Hence $\sigma / \theta$ is symmetric . Again let $x, y, z \in \mathcal{H}$, such that $(\theta(x), \theta(y)),(\theta(y), \theta(z)) \in \sigma / \theta$ and $(x, y),(y, z) \in$ $\sigma \Longrightarrow(x, z) \in \sigma$ due to the transitivity of $\sigma$. Which implies that $(\theta(x), \theta(y)) \in \sigma / \theta$. Hence $\sigma / \theta$ is transitive. Thus $\sigma / \theta$ is an equivalence relation. Now we have to show that, it is a regular. For it let $x, y, z \in \mathcal{H}$, such that $(\theta(x)) \sigma / \theta(\theta(y)) \Longrightarrow(x, y) \in \sigma \Longrightarrow x \sigma y \Longrightarrow(x z) \bar{\rho}(y z) \Longrightarrow\{\theta(\mu): \mu \in x z\} \overline{\sigma / \theta}\{\theta(\nu): \nu \in y z\}$. 
Which implies that $(\theta(x) \otimes \theta(z)) \overline{\sigma / \theta}(\theta(y) \otimes \theta(z))$ and similarly we can show that $(\theta(x)) \sigma / \theta(\theta(y)) \Longrightarrow$ $(\theta(z) \otimes \theta(x)) \overline{\sigma / \theta}(\theta(z) \otimes \theta(z))$. Hence $\sigma / \theta$ is regular relation on $H / \theta$.

Theorem 3.7. Let $\theta$ and $\sigma$ be two regular relations on an $\mathcal{H}_{v}-\mathcal{L} \mathcal{A}$-group $\mathcal{H}$ such that $\theta \subseteq \sigma$. Then $(\mathcal{H} / \theta) /(\sigma / \theta) \cong \mathcal{H} / \sigma$.

Proof: Let us define $\psi:(\mathcal{H} / \theta) /(\sigma / \theta) \longrightarrow \mathcal{H} / \sigma$ by $\psi(\sigma / \theta(\theta(x)))=\sigma(x) \forall x \in \mathcal{H}$. It is easy to show that this map is bijective. We only show that it is homomorphism. Suppose $x, y \in \mathcal{H}$, then

$$
\begin{aligned}
\psi(\sigma / \theta(\theta(x)) \otimes \sigma / \theta(\theta(y))) & =\psi(\{\sigma / \theta(\theta(z)): \theta(z) \in \theta(x) \otimes \theta(z)\}) \\
& =\psi(\{\sigma / \theta(\theta(z)): z \in x y\}) \\
& =\{\psi(\sigma / \theta(\theta(z)): z \in x y)\} \\
& =\{\sigma(z): z \in x y\}=\sigma(x) \sigma(y) \\
& =\psi(\sigma / \theta(\theta(x))) \otimes \psi(\sigma / \theta(\theta(y)))
\end{aligned}
$$

Hence $\psi$ is homomorphism. Thus $(\mathcal{H} / \theta) /(\sigma / \theta) \cong \mathcal{H} / \sigma$.

3.1. Construction of $\mathcal{H}_{v}-\mathcal{L} \mathcal{A}$-Groups: In this section we present the construction of $\mathcal{H}_{v}$ - $\mathcal{L} \mathcal{A}$-groups through any non-empty set having more than two elements.

Consider a finite set $\mathcal{H}$, such that $|\mathcal{H}|>2$. Define the hyperoperation $\circ$ on $\mathcal{H}$ as follows

$$
x_{i} \circ x_{j}=\left\{\begin{array}{cr}
x_{j} & \text { for } i=1 \\
x_{k} \text { for } j=1 \text { and } k \equiv 2-i \bmod |\mathcal{H}| \\
\mathcal{H} & \text { for } i=j, i \neq 1, j \neq 1 \\
x_{i} & \text { otherwise, for } i \prec j \text { or } i \succ j
\end{array}\right\}
$$

Then $\mathcal{H}$ under the hyperoperation $\circ$ form an $\mathcal{H}_{v}-\mathcal{L} \mathcal{A}$-group. The above construction can be explained with the help of an example.

Example: Let $\mathcal{H}=\left\{x_{1}, x_{2}, x_{3}\right\}$ be any set and define the binary hyperoperation $\circ$ defined above in the following cayley's table:

\begin{tabular}{|l|l|l|l|}
\hline$\circ$ & $x_{1,}$ & $x_{2}$ & $x_{3}$ \\
\hline$x_{1,}$ & $x_{1}$ & $x_{2}$ & $x_{3}$ \\
\hline$x_{2}$ & $x_{3}$ & $\mathcal{H}$ & $x_{2}$ \\
\hline$x_{3}$ & $x_{2}$ & $x_{3}$ & $\mathcal{H}$ \\
\hline
\end{tabular}

Then clearly $\mathcal{H}$ form an $\mathcal{H}_{v}-\mathcal{L} \mathcal{A}$-group. One can see that o satisfy the weak left invertive law with reproductive axiom, also $\circ$ is non-left invertive and non-associative i.e.

$$
\mathcal{H}=\left(x_{3} \circ x_{3}\right) \circ x_{2} \neq\left(x_{2} \circ x_{3}\right) \circ x_{3}=x_{2}
$$


and

$$
\mathcal{H}=\left(x_{2} \circ x_{2}\right) \circ x_{1} \neq x_{2} \circ\left(x_{2} \circ x_{1}\right)=x_{2}
$$

also it is not $W A S S$

$$
\left(x_{2} \circ x_{1}\right) \circ x_{1} \cap x_{2} \circ\left(x_{1} \circ x_{1}\right)=\phi .
$$

Hence $(\mathcal{H}, \circ)$ is an $\mathcal{H}_{v}$-LA-group. The result can easily be generalized to $n$ elements.

3.2. Chemical example of $\mathcal{H}_{v}-\mathcal{L} \mathcal{A}$ - group: Here in this section we utilize the newly defined structure namely $\mathcal{H}_{v}-\mathcal{L} \mathcal{A}$ - groups in applications. For this purpose we study chemical reactions. The best example of $\mathcal{H}_{v^{-}} \mathcal{L} \mathcal{A}$-group in chemical reaction is a redox reaction.

3.3. Redox reaction: The chemical reaction in which one specie loss the electron and other specie gain the electron. Oxidation mean loss of electron. Reduction mean gain of electron. The redox reaction is a vital for biochemical reaction and industrial process. The electron transfer in cell and oxidation of glucose in the human body are the example of redox reaction. The reaction between hydrogen and fluorine is an example of redox reaction i.e.

$$
\begin{aligned}
\mathcal{H}_{2}+F & \longrightarrow 2 \mathcal{H} F \\
\mathcal{H}_{2} & \longrightarrow 2 \mathcal{H}^{+}+2 e^{-}(\text {Oxidation }) \\
F_{2}+2 e^{-} & \longrightarrow 2 F \text { (Reduction) }
\end{aligned}
$$

Each half reaction has standard reduction potential $\left(E^{0}\right)$ which is equal to the potential difference at equilibrium under the standard condition of an electrochemical cell in which the cathode reaction is half reaction considered and anode is a standard hydrogen electrode (SHE). For the redox reaction, the potential of cell is defined as

$$
E^{\circ} \text { cell }=E^{\circ} \text { cathod }-E^{\circ} \text { anode }
$$

where $E^{\circ}$ anode is the standard potential at the anode and $E^{\circ}$ cathod is the standard potential at the cathode as given in the table of standard electrode potential. Now consider the redox reaction of $M n$

$$
\begin{aligned}
M n^{0}+2 M n^{+4}+2 M n^{+3} & \longrightarrow 3 M n^{+2}+2 M n^{+4} \\
M n^{0} & \longrightarrow M n^{+2}+M n^{+4}+2 e^{-}+2 M n^{+3}+2 M n^{+4}
\end{aligned}
$$

Manganese having a variable oxidation state of $0,+1,+2,+3,+4,+5,+6,+7$. If we take $M n^{0}, M n^{+4}, M n^{+3}, M n^{+4}$ together we will get pure redox reaction. The flow chart is given as 


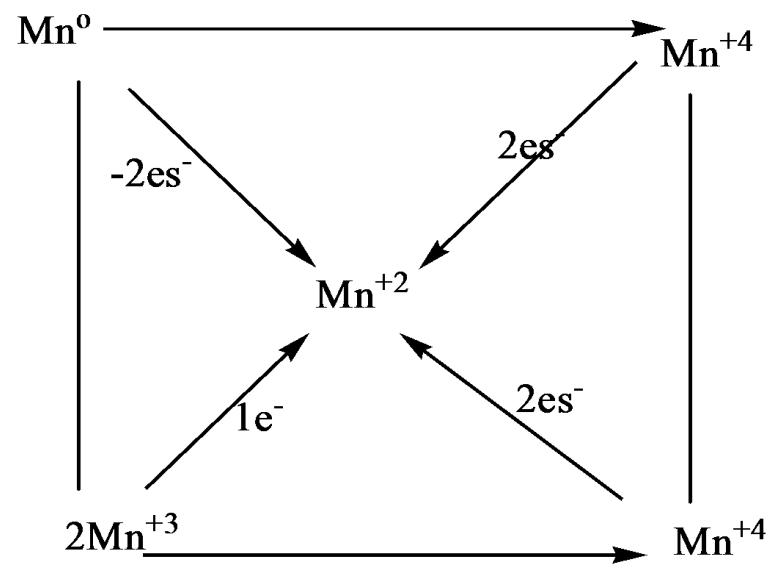

$M n$ species with different oxidation state react with themselves. All possible reactions are presented in the following table

\begin{tabular}{|l|l|l|l|l|l|}
\hline$\oplus$ & $M n^{0}$ & $M n^{+1}$ & $M n^{+2}$ & $M n^{+3}$ & $M n^{+4}$ \\
\hline$M n^{0}$ & $M n^{0}$ & $\left\{M n^{0}, M n^{+1}\right\}$ & $\left\{M n^{0}, M n^{+2}\right\}$ & $\left\{M n^{0}, M n^{+3}\right\}$ & $\left\{M n^{0}, M n^{+4}\right\}$ \\
\hline$M n^{+1}$ & $\left\{M n^{0}, M n^{+1}\right\}$ & $\left\{M n^{0}, M n^{+2}\right\}$ & $\left\{M n^{0}, M n^{+3}\right\}$ & $\left\{M n^{+2}\right\}$ & $\left\{M n^{+1}, M n^{+4}\right\}$ \\
\hline$M n^{+2}$ & $M n^{+1}$ & $\left\{M n^{0}, M n^{+3}\right\}$ & $\left\{M n^{+1}, M n^{+3}\right\}$ & $\left\{M n^{+1}, M n^{+4}\right\}$ & $\left\{M n^{+2}, M n^{+4}\right\}$ \\
\hline$M n^{+3}$ & $\left\{M n^{0}, M n^{+3}\right\}$ & $\left\{M n^{+1}, M n^{+3}\right\}$ & $\left\{M n^{+2}, M n^{+3}\right\}$ & $M n^{+3}$ & $\left\{M n^{+3}, M n^{+4}\right\}$ \\
\hline$M n^{+4}$ & $\left\{M n^{0}, M n^{+4}\right\}$ & $\left\{M n^{+1}, M n^{+4}\right\}$ & $\left\{M n^{+2}, M n^{+4}\right\}$ & $\left\{M n^{+3}, M n^{+4}\right\}$ & $M n^{+4}$ \\
\hline
\end{tabular}

The standard reduction potentials $\left(E^{0}\right)$ for conversion of each oxidation state to another are $E^{0}\left(M n^{+4} / M n^{+3}\right)=$ $+0.95, E^{0}\left(M n^{+3} / M n^{+2}\right)=+1.542, E^{0}\left(M n^{+2} / M n^{+1}\right)=-0.59, E^{0}\left(M n^{+1} / M n^{+0}\right)=0.296$. If we replace $M n^{0}=a, M n^{+1}=b, M n^{+2}=c, M n^{+3}=d, M n^{+4}=e$, then we obtain the following table

\begin{tabular}{|l|l|l|l|l|l|}
\hline$\oplus$ & $a$ & $b$ & $c$ & $d$ & $e$ \\
\hline$a$ & $\{a\}$ & $\{a, b\}$ & $\{a, c\}$ & $\{a, d\}$ & $\{a, e\}$ \\
\hline$b$ & $\{a, b\}$ & $\{a, c\}$ & $\{a, d\}$ & $\{c\}$ & $\{b, e\}$ \\
\hline$c$ & $\{a, c\}$ & $\{a, d\}$ & $\{b, d\}$ & $\{b, e\}$ & $\{c, e\}$ \\
\hline$d$ & $\{a, d\}$ & $\{b, d\}$ & $\{c, d\}$ & $\{d\}$ & $\{d, e\}$ \\
\hline$e$ & $\{a, e\}$ & $\{b, e\}$ & $\{c, e\}$ & $\{d, e\}$ & $\{e\}$ \\
\hline
\end{tabular}


As all elements of $\mathcal{H}$ satisfy the weak left invertive law with the reproductive axiom, but $\mathcal{H}$ do not satisfies the left invertive law, associative law and weak associative law

$$
\begin{aligned}
\{a, c\} & =(b \oplus b) \oplus a \neq(a \oplus b) \oplus b=\{a, b, c\}, \\
\{a, b, c, d\} & =(b \oplus b) \oplus c \neq b \oplus(b \oplus c)=\{a, b, c\}, \\
\text { and }(b \oplus d) \oplus d & =\{b, e\} \cap c=b \oplus(d \oplus d)=\phi .
\end{aligned}
$$

Hence $(\mathcal{H}, \oplus)$ is an $\mathcal{H}_{v}-\mathcal{L} \mathcal{A}$-group.

\section{Conclusion and Perspectives}

In this research, We have introduce a new class of $\mathcal{H}_{v}$ - $\mathcal{L} \mathcal{A}$-groups and investigated different types of homomorphisms of $\mathcal{H}_{v}-\mathcal{L} \mathcal{A}$-groups. Additionally, we construct the $\mathcal{H}_{v}-\mathcal{L} \mathcal{A}$-groups and applied our result to a chemical redox reaction. In the future work, we will apply our result to different kind of applications.

\section{REFERENCES}

[1] M. A. Kazim and M. Naseeruddin, On almost semigroups, Aligarh Bull. Math., 2 (1972), 1-7.

[2] Q. Mushtaq and S. M. Yusuf, On $\mathcal{L} \mathcal{A}$-semigroups, Aligarh Bull. Math., 8 (1978), 65-70.

[3] P. Holgate, Groupoids satisfying a simple invertive law, Math. Stud., 61(1-4) (1992), 101-106.

[4] J. R. Cho, J. Jezek and T. Kepka, Paramedial groupoids, Czechoslovak Math. J., 49(2) (1999), 277-290.

[5] M. Akram, N. Yaqoob and M. Khan, On (m, n)-ideals in $\mathcal{L} \mathcal{A}$-semigroups, Appl. Math. Sci., 7(44) (2013), $2187-2191$.

[6] M. Khan and N. Ahmad, Characterizations of left almost semigroups by their ideals, J. Adv. Res. Pure Math., 2(3) (2010), 61-73.

[7] P. V. Protic and N. Stevanovic, AG-test and some general properties of AbelGrassmann's groupoids, Pure Math. Appl., 6(4) (1995), 371-383.

[8] N. Stevanovic and P.V. Protic, Composition of Abel-Grassmann's 3-bands, Novi Sad J. Math., 34(2) (2004), $175-182$.

[9] Q. Mushtaq and S.M. Yusuf, On locally associative $\mathcal{L} \mathcal{A}$-semigroups, J. Nat. Sci. Math., 19(1) (1979), 57-62.

[10] Q. Mushtaq and M. S. Kamran, Left almost group, Proc. Pak. Acad Sci., 33 (1996), 12

[11] F. Marty, Sur une generalization de la notion de groupe, 8iem Congres des Mathematicians Scandinaves Tenua Stockholm, (1934) 45-49.

[12] P. Corsini, Prolegomena of hypergroup theory, Aviani Editore, (1993).

[13] T. Vougiouklis, Hyperstructures and their representations, Hadronic Press,Palm Harbor, Flarida, USA, (1994).

[14] P. Corsini and V. Leoreanu, Applications of hyperstructure theory, Kluwer Academic, (2003).

[15] K. Hila and J. Dine, On hyperideals in left almost semihypergroups, ISRN Algebra, 2011 (2011), Article ID 953124.

[16] N. Yaqoob, P. Corsini and F. Yousafzai, On intra-regular left almost semihypergroups with pure left identity, J. Math., 2013 (2013), Article ID 510790.

[17] T. Vougiouklis, The fundamental relation in hyperrings. The general hyperfield, Algebraic Hyperstructures and Applications, Proceedings of the Fourth International Congress, (1991), 203-211.

[18] T. Vougiouklis, A new class of hyperstructures, Journal of Combinatorics, Inform. Syst. Sci., 20 (1995), $229-235$.

[19] T. Vougiouklis, $\partial$-operations and $\mathcal{H}_{v}$-fields, Acta Math. Sin. (Engl. Ser.), 24(7) (2008), 1067-1078. 
[20] T. Vougiouklis, The h/v-structures, Algebraic Hyperstructures and Applications, Taru Publications, New Delhi, (2004), 115-123.

[21] S. Spartalis, On $\mathcal{H}_{v}$-semigroups, Italian J. Pure Appl. Math., 11 (2002), 165-174.

[22] S. Spartalis, On the number of $\mathcal{H}_{v}$-rings with P-hyperoperations, Discr. Math., 155 (1996), 225-231.

[23] S. Spartalis, On reversible $\mathcal{H}_{v}$-group, Algebr. Hyperstruct. Appl., (1994) 163-170.

[24] S. Spartalis, Quoitients of P- $\mathcal{H}_{v}$-rings, New Front. Hyperstruct., (1996) 167-176.

[25] S. Spartalis and T. Vougiouklis, The fundamental relations on $\mathcal{H}_{v}$-rings, Riv. Mat. Pura Appl., 7 (1994), 7-20.

[26] M. Gulistan, N. Yaqoob and M. Shahzad, A Note On $\mathcal{H}_{v}-\mathcal{L} \mathcal{A}$-semigroup U.P.B. Sci. Bull., Series A, 77 (3) (2015), 93-106

[27] B. Davvaz, Weak algebraic hyperstructures as a model for interpretation of chemical reactions, Iran. J. Math. Chem. 7 (2) (2016), 267-283.

[28] B. Davvaz, A. Dehghan Nezhad, A. Benvidi, Chemical hyperalgebra: Dismutation reactions, MATCH Commun. Math. Comput. Chem. 67 (2012), 55-63.

[29] B. Davvaz, A. D. Nezad and A. Benvidi, Chain reactions as experimental examples of ternary algebraic hyperstructures, MATCH Commun. Math. Comput. Chem. 65 (2) (2011), 491-499. 